\title{
Identification of biological processes and signaling pathways for the knockout of REV-ERB in mouse brain
}

Jing Li, Wei Wang, Hanming Gu ${ }^{1}$

${ }^{1}$ SHU-UTS SILC School, Shanghai University, Shanghai, China

*Corresponding author: Hanming Gu, SHU-UTS SILC School, Shanghai University, Shanghai, China

laygmp@gmail.com 


\section{Abstract}

REV-ERB is an orphan nuclear receptor that is widely expressed in the brain and inhibits transcriptional activities. A variety of genes affect the activity and expression of REV-ERB. In this study, our objective is to identify significant signaling pathways and biological processes in the knockout of the REV-ERB mouse brain. The GSE152919 dataset was originally created by using the Illumina HiSeq 4000 (Mus musculus). The KEGG and GO analyses suggested that biological processes "PPAR signaling", "Hippo signaling", and "Hypertrophic cardiomyopathy (HCM)" are mostly affected in the knockout of REV-ERB. Furthermore, we identified a number of genes according to the PPI network including NPAS2, CRY2, BMAL1, and CRY1 which were involved in the lack of REV-ERB in the brain. Therefore, our study provides further insights into the study of circadian clocks.

\section{Introduction}

The circadian clocks control the behavior and physiology of living organisms according to the external environment ${ }^{1,2}$. The core circadian clocks regulate transcriptional and physiological rhythms which form a transcriptional-translational feedback loop ${ }^{3}$. The core circadian clocks contain transcriptional activators Bmal1/CIOCK which activates their repressor proteins such as PER, CRY, and REV-ERB ${ }^{4}$. The circadian clocks control various cellular processes such as metabolism, inflammation, and mitochondrial homeostasis ${ }^{5-9}$. Clocks' function and cycles of energy metabolism are closely and reciprocally linked ${ }^{10-12}$. The disruption of clocks leads to metabolic diseases such as type 2 diabetes and heart diseases ${ }^{13}$.

$\mathrm{REV}-\mathrm{ERBa}$ is a nuclear reporter protein that is directly mediated by BMAL1 ${ }^{14}$. REV$\mathrm{ERBa}$ is also a transcriptional repressor that restrains Bmal1 expression and potential downstream genes at specific sites within the genome ${ }^{15}$. Given that REV-ERBa locates in the nucleus, it becomes a potential drug target that can be regulated by smallmolecule agonists and antagonists ${ }^{16}$. Recent reports showed that REV-ERBa is one of the key regulators in mediating the energy metabolism ${ }^{17}$. The REV-ERBa mice depicted remarkable changes in the homeostasis of carbohydrate and lipid, which displayed an up-regulation of lipid accumulation and storage ${ }^{18}$. REV-ERBa is regulated by 
BMAL1/CLOCK heterodimers through transactivation and posttranslational protein degradation ${ }^{17}$. REV-ERBa indicates circadian rhythmic activity, which competes and binds with the RORE sites (AGGTCA hexamer with a 5' A/T-rich sequence) of ROR proteins ${ }^{19}$. REV-ERBa knockout mice exhibited significantly changed cortical restingstate functional connectivity, which was found in neurodegenerative models ${ }^{20}$.

In our study, we evaluated the effects of knockout of REV-ERB in the suprachiasmatic nucleus (SCN) during the nighttime by analyzing the RNA sequence data. We identified a number of DEGs and the potential affected biological processes. We also performed the gene functional enrichment and constructed the protein-protein interaction (PPI) network for finding the potential interacting proteins. These important genes and biological processes could provide efficient guidance on drug development.

\section{Methods}

\section{Data resources}

Gene dataset GSE152919 was collected from the GEO database. The data was created by using the Illumina HiSeq 4000 (Mus musculus) (Institute for Diabetes, Obesity, and Metabolism, University of Pennsylvania Perelman School of Medicine, Philadelphia, PA19104-5160, US). The analyzed dataset includes 5 WT and 4 REVERB KO at CT16.

\section{Data acquisition and preprocessing}

The data were conducted by the $R$ package. We used a classical t-test to identify DEGs with $\mathrm{P}<.05$ and fold change $\geq 1.5$ as being statistically significant ${ }^{21,22}$.

The Kyoto Encyclopedia of Genes and Genomes (KEGG) and Gene Ontology (GO) analyses

KEGG and GO analyses of DEGs in this study were conducted by the Database for Annotation, Visualization, and Integrated Discovery (DAVID) (http://david.ncifcrf.gov/). $\mathrm{P}<.05$ and gene counts $>10$ were considered statistically significant.

Protein-protein interaction (PPI) networks 
The Molecular Complex Detection (MCODE) was used to construct the PPI networks. The significant modules were created from constructed PPI networks. The pathway enrichment analyses were performed by using Reactome (https://reactome.org/), and $\mathrm{P}<0.05$ was used as the cutoff criterion.

\section{Results}

\section{Identification of DEGs in WT and REV-ERB KO}

Since REV-ERB showed higher expression during the night in comparison with daytime, we analyzed the DEGs from the WT and REV-ERB KO mice at Zeitgeber times (ZT) $16^{23}$. A total of 228 genes were identified to be differentially expressed with the threshold of $P<0.05$. The up- and down-regulated genes for WT and REV-ERB KO samples were depicted by the heatmap and volcano plot (Figure 1). Among them, the top ten DEGs were selected and listed in Table 1.

\section{Enrichment analysis of DEGs in WT and REV-ERB KO}

To further understand the biological roles of REV-ERB, we performed KEGG and GO enrichment analysis (Figure 2). The top five significant KEGG pathways were analyzed including " Circadian rhythm", "Oxytocin signaling pathway", "Hippo signaling pathway", "Hypertrophic cardiomyopathy (HCM)", and "PPAR signaling pathway". We identified the top ten MF categories of GO including "Nucleoside-triphosphatase regulator activity", "Enzyme activator activity", "GTPase regulator activity", "Metal ion transmembrane transporter activity", "amide binding", "GTPase activator activity", "Calcium ion transmembrane transporter activity", "Voltage-gated cation channel activity", "calcium channel activity", and "Voltage-gated calcium channel activity".

Then, we identified the top ten BP categories of GO including "Synapse organization", "Calcium ion homeostasis", "cellular calcium ion homeostasis", "Urogenital system development", "Extracellular matrix organization", "Extracellular structure organization", "External encapsulating structure organization", "vascular process in circulatory system", "negative regulation of cell development", and "synapse assembly". We also identified the top ten CC of GO including "collagen-containing extracellular matrix", "Intrinsic component of synaptic membrane", "cation channel complex", "extrinsic component of 
plasma membrane", "cytoplasmic side of membrane”, "cell-substrate junction”, "integral component of postsynaptic membrane", "sarcolemma", "ndoplasmic reticulum protein-containing complex", "basement membrane".

\section{PPI network analysis in WT and REV-ERB KO}

The PPI network was created to explore the relationships of DEGs affected by REVERB. The criterion of combined score $>0.4$ was set to construct the PPI by using the 127 nodes and 201 edges. The top ten genes with the highest degree scores are shown in Table 2. The top two significant modules were selected to depict the functional annotation (Figure 3). We also analyzed the DEGs and PPI with the Reactome analysis tools.

The Reactome map showed the most biological functions affected by the knockout of REV-ERB (Figure 4). We also identified the top ten signaling pathways including "Circadian Clock", "BMAL1:CLOCK, NPAS2 activates circadian gene expression", "Transcriptional activation of cell cycle inhibitor p21", "Transcriptional activation of p53 responsive genes “, "Heme signaling”, "TFAP2 (AP-2) family regulates transcription of cell cycle factors", "TP53 Regulates Transcription of Genes Involved in G1 Cell Cycle Arrest", "Sodium-coupled phosphate cotransporters", "Extracellular matrix organization" , and "RUNX3 regulates CDKN1A transcription" (Supplemental Table S1).

\section{Discussion}

As one of the transcriptional repressors, REV-ERB can inhibit gene transcription by recruiting co-factors nuclear receptor co? repressor 1 (NCOR1) and histone deacetylase 3 (HDAC3 $^{24}$. Given that REV-ERBa regulates the clock and metabolic genes, it is proposed as a drug target for treating metabolic syndromes such as obesity and diabetes $^{25}$. Recent studies showed various roles of REV-ERBa including inflammatory diseases, cancers, and heart diseases ${ }^{25}$. Moreover, REV-ERBa and its ligands have been considered valuable pharmacological molecules ${ }^{16}$.

REV-ERB is important for the development of metabolic diseases ${ }^{16}$. In our study, the KEGG analysis showed PPAR signaling, Hippo signaling, and Hypertrophic cardiomyopathy $(\mathrm{HCM})$ are the most affected biological processes during the knockout 
of REV-ERB at night. In the study by Coralie Fontaine, REV-ERBa can activate the PPAR and further drive the adipocyte differentiation ${ }^{26}$. Protein modification is an important step in regulating molecular activity under physiological and pathological conditions. REV-ERBa facilitates cytosolic and nuclear protein O-GlcNAcylation that can change the activity of YAP in the hippo signaling pathway ${ }^{27}$. Moreover, Lilei et al found REV-ERBa inhibits heart failure by repressing the transcription ${ }^{28}$. These findings are supported by our study. In the study of GO (BP analysis), we found that the nucleosidetriphosphatase regulator activity was the most affected process during the deficiency of REV-ERB. The core circadian clocks such as BMAL1 and CLOCK were located in the nucleus and regulated the transcriptional activities of target genes including NF-KB and RANKL to further regulate the downstream signaling pathways ${ }^{29-31}$. REV-ERBa was also located in the nucleus and repressed the activity of BMAL $1^{32}$. It is suggested that REV-ERBa may affect the transcriptional activities through BMAL1. Interestingly, we also found that the knockout of REV-ERB can affect GPCR signaling pathways. GPCR and RGS signaling pathways play key roles in mediating the physiological and pathological processes such as metabolism ${ }^{33,34}$, inflammation ${ }^{35-39}$, and tumorigenesis ${ }^{40,}$ 41. It was found that REV-ERB forms complexes with NR2E3 to further regulate the expression of Guanine nucleotide-binding protein 1 (Gnat1) ${ }^{42}$. We also found that REVERB KO affects the synapse organization and assembly. Supportively, Tianpeng et al found the disorder of REV-ERBa inhibits GABAergic function and drives epileptic seizures in preclinical models ${ }^{43}$. Moreover, REV-ERB regulates the complement expression and microglial synaptic phagocytosis ${ }^{44}$.

In our study, the PPI analysis identified a number of critical genes that may affect the biological processes in REV-ERB KO mice. NPAS2 is highly expressed in the brain and can control the anxiety-like behavior and GABAA receptors ${ }^{45}$. Cry2 is one of the core components of circadian clocks which has been linked to depression in patients ${ }^{46}$. BMAL1 is a basic helix loop helix transcription factor that binds with its partner CLOCK or NPAS2 to control the circadian oscillations ${ }^{47}$. The circadian controlled pathways include PER, CRY, NR1D1, and other genes that underlie circadian oscillation of ER stress, molecule activity, and oxidant defenses ${ }^{48-51}$. Cry1 is also highly expressed in the brain that associates with PER, which leads to the inhibition of CLOCK-BMAL1 to 
further control the clock-controlled genes ${ }^{52}$. As a core circadian gene, Per3 can regulate the embryonic development of the cerebral cortex ${ }^{53}$. Bhlhe41 is required for the competitive fitness of alveolar macrophages and the knockout of Bhlhe41 inhibits the proliferation of macrophages ${ }^{54}$. Hui et al found NR1D2 can promote the progression of liver cancer by regulating the epithelial transition ${ }^{55}$. As a survival factor, NFIL3 can inhibit the FOXO-regulated gene expression in cancer ${ }^{56}$. TEF (thyrotroph embryonic factor) is an important factor of the PAR bZip members, which is expressed in the brain and is relevant to intractable epilepsy ${ }^{57,58}$.

In summary, our study provided the insight into the knockout of REV-ERB in mouse brains. The PPAR signaling, Hippo signaling, and Hypertrophic cardiomyopathy (HCM) are the significant biological processes during the deficiency of REV-ERB in the brain. Our future studies will explore the upstream and downstream of the important processes. Our study may facilitate the research on circadian rhythms.

\section{Author Contributions}

Jing Li, Wei Wang: Methodology and Writing. Hanming Gu: Conceptualization, Methodology, Writing- Reviewing and Editing.

\section{Funding}

This work was not supported by any funding.

\section{Declarations of interest}

There is no conflict of interest to declare. 


\section{Figure Legends}

A

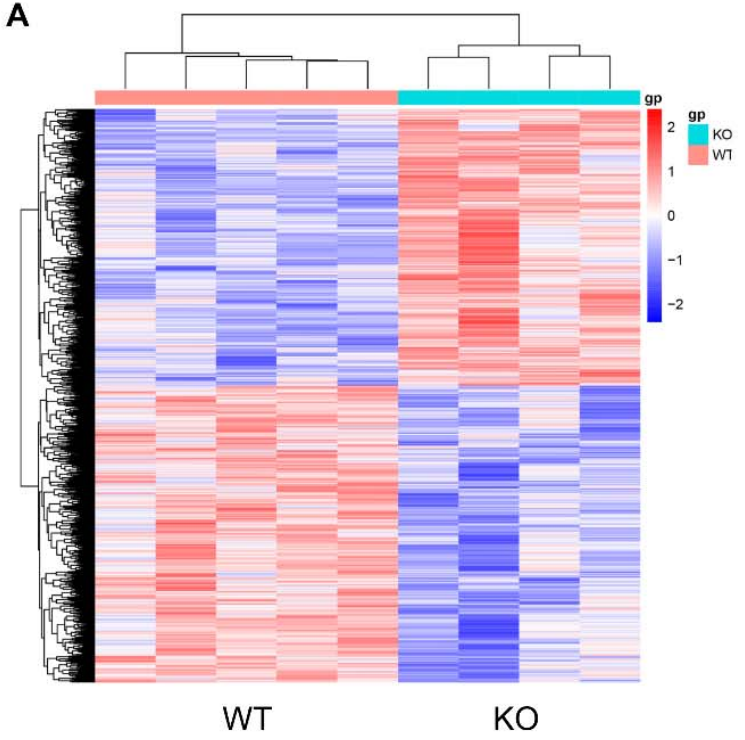

B

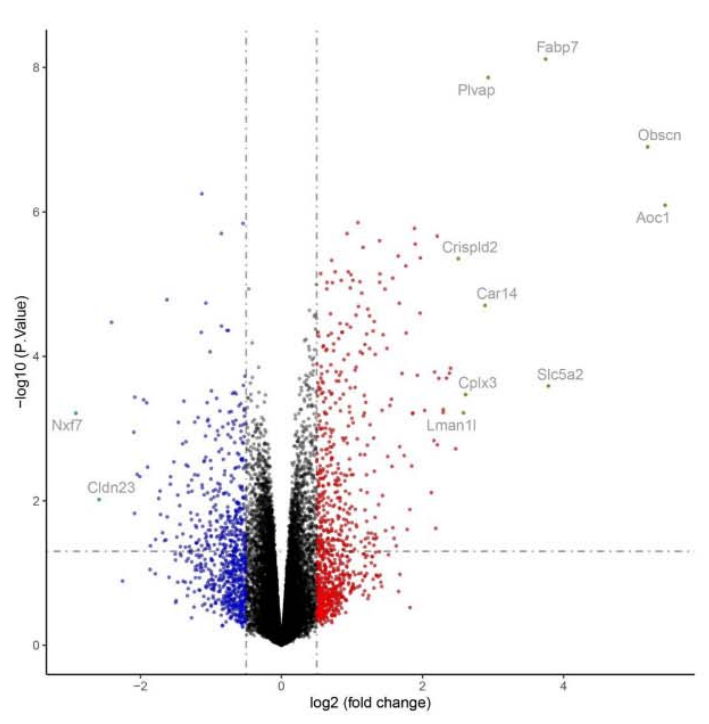

Figure 1. Heatmap and volcano plot between WT and REV-ERB KO

(A) Heatmap of significant DEGs between WT and REV-ERB KO. Regularized matrix was generated using the $R$ package. Significant DEGs were used to create the heatmap. (B) Volcano plot for DEGs between WT and REV-ERB KO. The most significant genes are highlighted grey dots and gene symbols marked. 


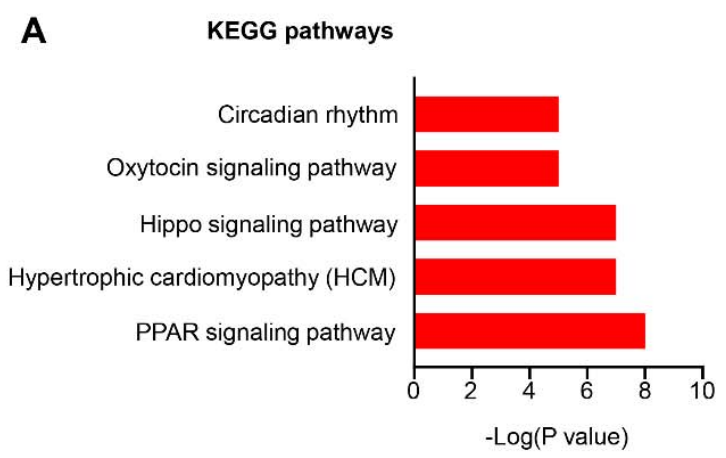

B

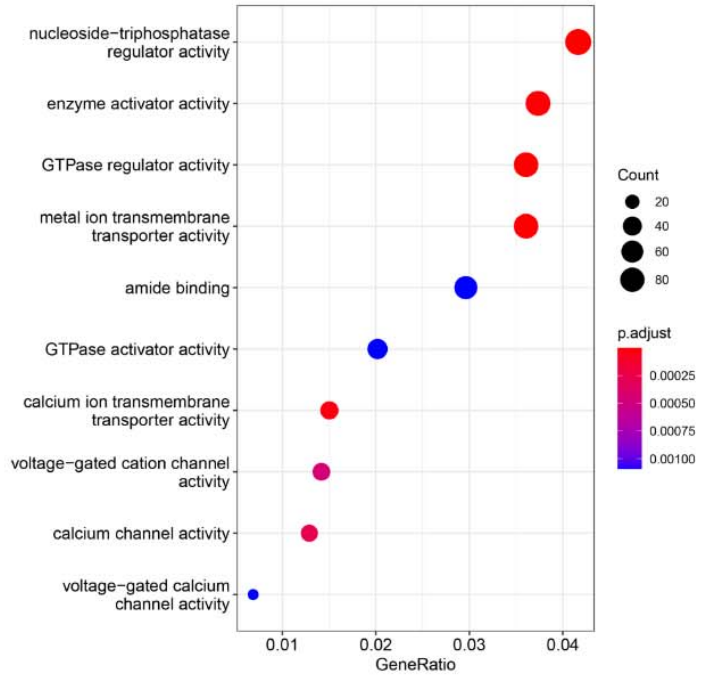

C

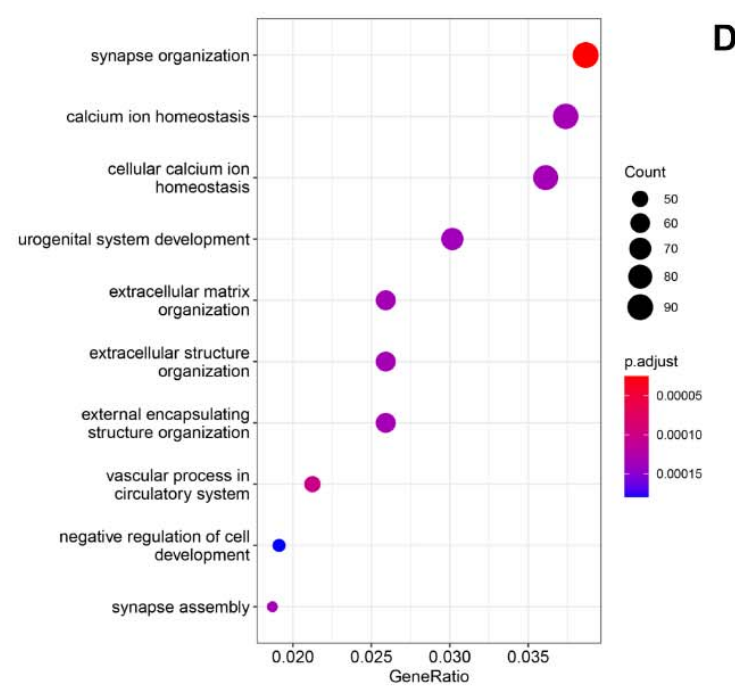

D

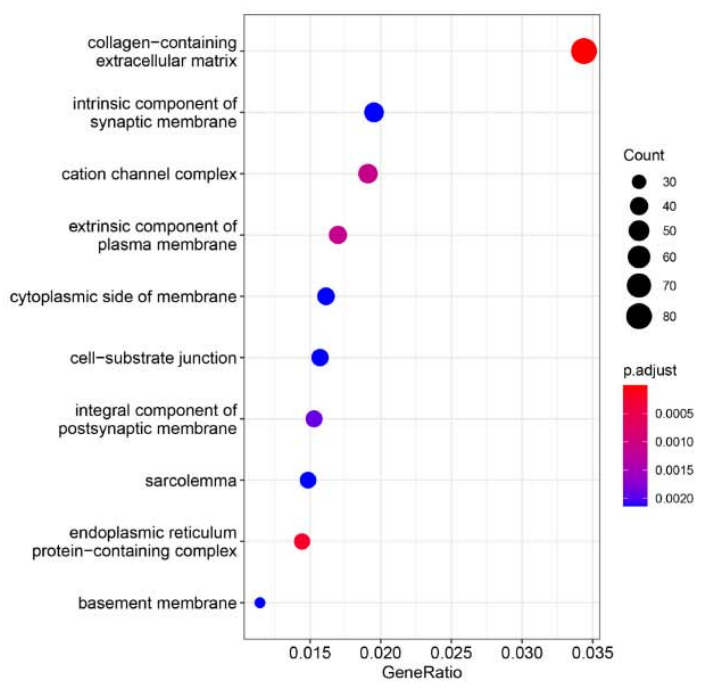

Figure 2. KEGG and GO analyses of DEGs between WT and REV-ERB KO

(A) KEGG analysis was performed by DAVID online tool. The significant terms were depicted. (B) Different colors represent biological processes (BP). (C) The molecular functions (MF) were analyzed by DAVID. (D) The cellular components (CC) were performed by DAVID. 
A

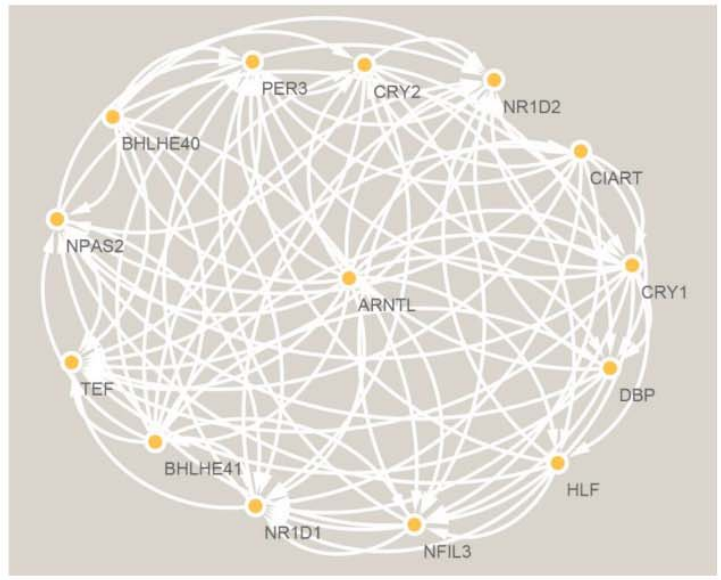

B

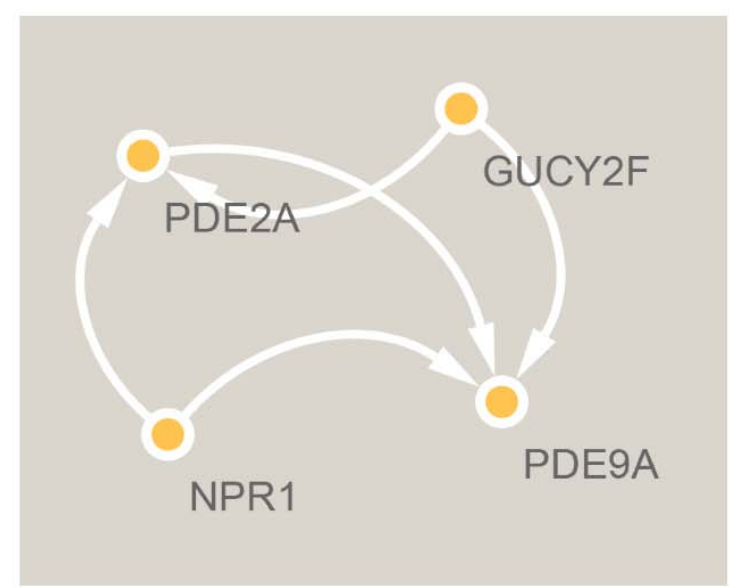

Figure 3. The PPI network analysis of DEGs between WT and REV-ERB KO The 227 DEGs were input into the STRING database for PPI network analysis. Cluster 1 (A) and cluster 2 (B) were constructed by MCODE in Cytoscape. 
bioRxiv preprint doi: https://doi.org/10.1101/2021.11.22.469579; this version posted November 22, 2021. The copyright holder for this preprint (which was not certified by peer review) is the author/funder, who has granted bioRxiv a license to display the preprint in perpetuity. It is made available under aCC-BY-NC-ND 4.0 International license.

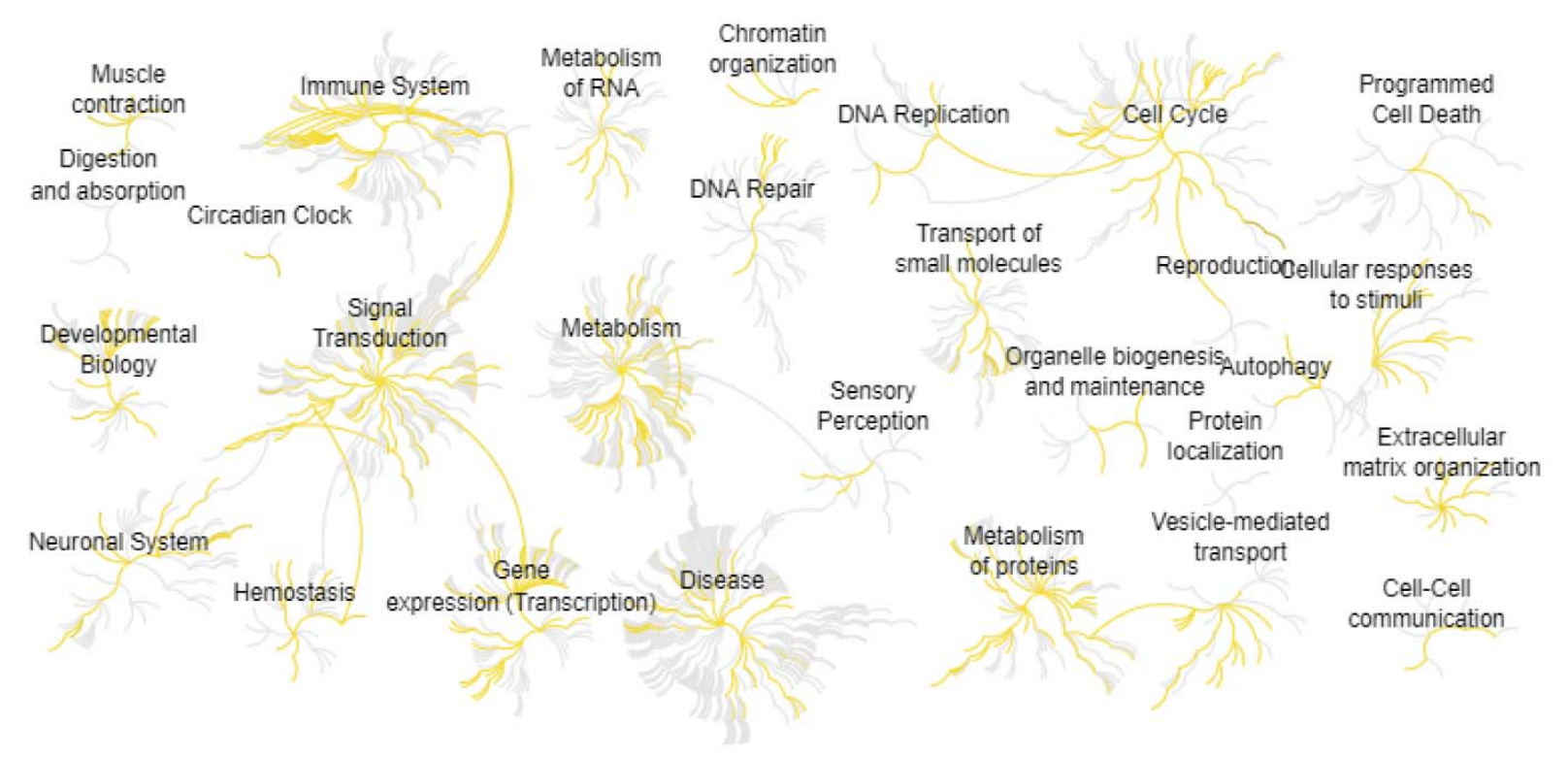

Figure 4. Reactome diagram representation of the significant biological processes of the protein elements identified between WT and REV-ERB KO 


\begin{tabular}{llll}
\multicolumn{1}{l}{ Table 1 } & & & \\
\hline \multicolumn{1}{l}{ Entrez gene } & Gene Symble & Fold-change & Regulation \\
\hline \multicolumn{1}{l}{ Top 10 down-regulated DEGs } & & \\
170722 & Nxf7 & -2.914409539 & Down \\
84063 & Kirrel2 & -2.408625265 & Down \\
255349 & Tmem211 & -2.079681186 & Down \\
74879 & 4930461G14Rik & -1.953485978 & Down \\
51458 & Rhcg & -1.909884329 & Down \\
64833 & Acot10 & -1.623019882 & Down \\
102466639 & Mir6385 & -1.383534957 & Down \\
73040 & 2900052N01Rik & -1.135864344 & Down \\
4240 & Mfge8 & -1.127675784 & Down \\
70393 & 2210416O15Rik & -1.0711531 & Down \\
Top 10 up-regulated DEGs & & \\
26 & Aoc1 & 5.441097014 & up \\
84033 & Obscn & 5.192832086 & up \\
6524 & Slc5a2 & 3.785831939 & up \\
2173 & Fabp7 & 3.745998775 & up \\
83483 & Plvap & 2.931300223 & up \\
23831 & Car14 & 2.885290644 & up \\
594855 & Cplx3 & 2.611357879 & up \\
79748 & Lman1l & 2.581577124 & up \\
83716 & Crispld2 & 2.508233633 & up \\
329271 & C230024C17Rik & 2.398221442 & up \\
\hline
\end{tabular}


Table 2. Top ten genes demonstrated by connectivity degree in the PPI network

\begin{tabular}{lll}
\hline Gene Symbol & Gene title & Degree \\
\hline NPAS2 & Neuronal PAS domain protein 2 & 16 \\
CRY2 & $\begin{array}{l}\text { Cryptochrome circadian regulator 2 } \\
\text { aryl hydrocarbon receptor nuclear }\end{array}$ & 16 \\
ARNTL & translocator like & 14 \\
CRY1 & cryptochrome circadian regulator 1 & 14 \\
PER3 & period circadian regulator 3 & 13 \\
BHLHE41 & basic helix-loop-helix family member e41 & 13 \\
& nuclear receptor subfamily 1 group D & \\
NR1D2 & member 2 & 13 \\
NR1D1 & nuclear receptor subfamily 1 group D & 13 \\
NFIL3 & member 1 & 12 \\
TEF & nuclear factor, interleukin 3 regulated & 12 \\
\hline
\end{tabular}




\section{References}

[1] Wager-Smith K, Kay SA: Circadian rhythm genetics: from flies to mice to humans. Nat Genet 2000, 26:23-7.

[2] Yuan G, Hua B, Cai T, Xu L, Li E, Huang Y, Sun N, Yan Z, Lu C, Qian R: Clock mediates liver senescence by controlling ER stress. Aging 2017, 9:2647-65.

[3] Benito J, Zheng H, Ng FS, Hardin PE: Transcriptional feedback loop regulation, function, and ontogeny in Drosophila. Cold Spring Harb Symp Quant Biol 2007, 72:437-44.

[4] Takahashi JS: Transcriptional architecture of the mammalian circadian clock. Nat Rev Genet 2017, 18:164-79.

[5] Marcheva B, Ramsey KM, Peek CB, Affinati A, Maury E, Bass J: Circadian clocks and metabolism. Handb Exp Pharmacol 2013:127-55.

[6] Xu L, Cheng Q, Hua B, Cai T, Lin J, Yuan G, Yan Z, Li X, Sun N, Lu C, Qian R: Circadian gene Clock regulates mitochondrial morphology and functions by posttranscriptional way. bioRxiv 2018:365452.

[7] Zhu Z, Hua B, Xu L, Yuan G, Li E, Li X, Sun N, Yan Z, Lu C, Qian R: CLOCK promotes 3T3-L1 cell proliferation via Wnt signaling. IUBMB Life 2016, 68:557-68.

[8] Cai T, Hua B, Luo D, Xu L, Cheng Q, Yuan G, Yan Z, Sun N, Hua L, Lu C: The circadian protein CLOCK regulates cell metabolism via the mitochondrial carrier SLC25A10. Biochim Biophys Acta Mol Cell Res 2019, 1866:1310-21.

[9] Yuan G, Hua B, Yang Y, Xu L, Cai T, Sun N, Yan Z, Lu C, Qian R: The Circadian Gene Clock Regulates Bone Formation Via PDIA3. J Bone Miner Res 2017, 32:861-71.

[10] Ma D, Li S, Molusky MM, Lin JD: Circadian autophagy rhythm: a link between clock and metabolism? Trends Endocrinol Metab 2012, 23:319-25.

[11] Zhu Z, Hua B, Shang Z, Yuan G, Xu L, Li E, Li X, Sun N, Yan Z, Qian R, Lu C: Altered Clock and Lipid Metabolism-Related Genes in Atherosclerotic Mice Kept with Abnormal Lighting Condition. Biomed Res Int 2016, 2016:5438589.

[12] Zhu Z, Xu L, Cai T, Yuan G, Sun N, Lu C, Qian R: Clock represses preadipocytes adipogenesis via GILZ. J Cell Physiol 2018, 233:6028-40.

[13] Zimmet P, Alberti K, Stern N, Bilu C, El-Osta A, Einat H, Kronfeld-Schor N: The Circadian Syndrome: is the Metabolic Syndrome and much more! J Intern Med 2019, 286:181-91.

[14] Chatterjee S, Yin H, Li W, Lee J, Yechoor VK, Ma K: The Nuclear Receptor and Clock Repressor Reverbalpha Suppresses Myogenesis. Sci Rep 2019, 9:4585.

[15] Everett L, Lazar MA: Nuclear receptor Rev-erbalpha: up, down, and all around. Trends Endocrinol Metab 2014, 25:586-92.

[16] Kojetin DJ, Burris TP: REV-ERB and ROR nuclear receptors as drug targets. Nat Rev Drug Discov 2014, 13:197-216.

[17] Duez H, Staels B: Rev-erb-alpha: an integrator of circadian rhythms and metabolism. J Appl Physiol (1985) 2009, 107:1972-80.

[18] Le Martelot G, Claudel T, Gatfield D, Schaad O, Kornmann B, Lo Sasso G, Moschetta A, Schibler U: REV-ERBalpha participates in circadian SREBP signaling and bile acid homeostasis. PLoS Biol 2009, 7:e1000181.

[19] Yin L, Wu N, Lazar MA: Nuclear receptor Rev-erbalpha: a heme receptor that coordinates circadian rhythm and metabolism. Nucl Recept Signal 2010, 8:e001.

[20] Griffin P, Dimitry JM, Sheehan PW, Lananna BV, Guo C, Robinette ML, Hayes ME, Cedeno MR, Nadarajah CJ, Ezerskiy LA, Colonna M, Zhang J, Bauer AQ, Burris TP, Musiek ES: Circadian clock protein Rev-erbalpha regulates neuroinflammation. Proc Natl Acad Sci U S A 2019, 116:5102-7.

[21] Gu H, Wang W, Yuan G: Identification of biomarkers and candidate inhibitors for multiple myeloma. bioRxiv 2021:2021.02.25.432847. 
[22] Hanming G, Gongsheng Y: Identification of key genes and pathways in the hPSC-derived lungs infected by the SARS-CoV-2. Research Square 2021.

[23] Verlande A, Chun SK, Goodson MO, Fortin BM, Bae H, Jang C, Masri S: Glucagon regulates the stability of REV-ERBalpha to modulate hepatic glucose production in a model of lung cancer-associated cachexia. Sci Adv 2021, 7.

[24] Mottis A, Mouchiroud L, Auwerx J: Emerging roles of the corepressors NCoR1 and SMRT in homeostasis. Genes Dev 2013, 27:819-35.

[25] Wang S, Li F, Lin Y, Wu B: Targeting REV-ERBalpha for therapeutic purposes: promises and challenges. Theranostics 2020, 10:4168-82.

[26] Fontaine C, Dubois G, Duguay Y, Helledie T, Vu-Dac N, Gervois P, Soncin F, Mandrup S, Fruchart JC, Fruchart-Najib J, Staels B: The orphan nuclear receptor Rev-Erbalpha is a peroxisome proliferatoractivated receptor (PPAR) gamma target gene and promotes PPARgamma-induced adipocyte differentiation. J Biol Chem 2003, 278:37672-80.

[27] Kim E, Kang JG, Kang MJ, Park JH, Kim YJ, Kweon TH, Lee HW, Jho EH, Lee YH, Kim SI, Yi EC, Park HW, Yang WH, Cho JW: O-GICNAcylation on LATS2 disrupts the Hippo pathway by inhibiting its activity. Proc Natl Acad Sci U S A 2020, 117:14259-69.

[28] Zhang L, Zhang R, Tien CL, Chan RE, Sugi K, Fu C, Griffin AC, Shen Y, Burris TP, Liao X, Jain MK: REVERBalpha ameliorates heart failure through transcription repression. JCI Insight 2017, 2.

[29] Maury E: Off the Clock: From Circadian Disruption to Metabolic Disease. Int J Mol Sci 2019, 20.

[30] Yuan G, Xu L, Cai T, Hua B, Sun N, Yan Z, Lu C, Qian R: Clock mutant promotes osteoarthritis by inhibiting the acetylation of NFkappaB. Osteoarthritis Cartilage 2019, 27:922-31.

[31] Yuan G: Identification of biomarkers and pathways of mitochondria in sepsis patients. bioRxiv 2021:2021.03.29.437586.

[32] Ikeda R, Tsuchiya Y, Koike N, Umemura Y, Inokawa H, Ono R, Inoue M, Sasawaki Y, Grieten T, Okubo N, Ikoma K, Fujiwara H, Kubo T, Yagita K: REV-ERBalpha and REV-ERBbeta function as key factors regulating Mammalian Circadian Output. Sci Rep 2019, 9:10171.

[33] Yuan G, Yang S, Liu M, Yang S: RGS12 is required for the maintenance of mitochondrial function during skeletal development. Cell Discov 2020, 6:59.

[34] Yuan G, Yang S, Yang S: Macrophage RGS12 contributes to osteoarthritis pathogenesis through enhancing the ubiquitination. Genes \& Diseases 2021.

[35] Doyen PJ, Vergouts M, Pochet A, Desmet N, van Neerven S, Brook G, Hermans E: Inflammationassociated regulation of RGS in astrocytes and putative implication in neuropathic pain. J Neuroinflammation 2017, 14:209.

[36] Yuan G, Yang S, Yang S, Ng A, Oursler MJ: RGS12 is a critical proinflammatory factor in the pathogenesis of inflammatory arthritis via acting in Cox2-RGS12-NF kappa B pathway activation loop. J Bone Miner Res: WILEY 111 RIVER ST, HOBOKEN 07030-5774, NJ USA, 2019. pp. 147-.

[37] Yuan G, Yang S, Ng A, Fu C, Oursler MJ, Xing L, Yang S: RGS12 Is a Novel Critical NF-kappaB Activator in Inflammatory Arthritis. iScience 2020, 23:101172.

[38] Xie Z, Chan EC, Druey KM: R4 Regulator of G Protein Signaling (RGS) Proteins in Inflammation and Immunity. AAPS J 2016, 18:294-304.

[39] Yuan G, Yang S, Gautam M, Luo W, Yang S: Macrophage regulator of G-protein signaling 12 contributes to inflammatory pain hypersensitivity. Ann Transl Med 2021, 9:448.

[40] Cao X, Qin J, Xie Y, Khan O, Dowd F, Scofield M, Lin MF, Tu Y: Regulator of G-protein signaling 2 (RGS2) inhibits androgen-independent activation of androgen receptor in prostate cancer cells. Oncogene 2006, 25:3719-34.

[41] Fu C, Yuan G, Yang ST, Zhang D, Yang S: RGS12 Represses Oral Cancer via the Phosphorylation and SUMOylation of PTEN. J Dent Res 2020:22034520972095. 
[42] Cheng H, Khanna H, Oh EC, Hicks D, Mitton KP, Swaroop A: Photoreceptor-specific nuclear receptor NR2E3 functions as a transcriptional activator in rod photoreceptors. Hum Mol Genet 2004, 13:1563-75.

[43] Zhang T, Yu F, Xu H, Chen M, Chen X, Guo L, Zhou C, Xu Y, Wang F, Yu J, Wu B: Dysregulation of REVERBalpha impairs GABAergic function and promotes epileptic seizures in preclinical models. Nat Commun 2021, 12:1216.

[44] Griffin P, Sheehan PW, Dimitry JM, Guo C, Kanan MF, Lee J, Zhang J, Musiek ES: REV-ERBalpha mediates complement expression and diurnal regulation of microglial synaptic phagocytosis. Elife 2020, 9.

[45] Ozburn AR, Kern J, Parekh PK, Logan RW, Liu Z, Falcon E, Becker-Krail D, Purohit K, Edgar NM, Huang Y, McClung CA: NPAS2 Regulation of Anxiety-Like Behavior and GABAA Receptors. Front Mol Neurosci 2017, 10:360.

[46] Savalli G, Diao W, Berger S, Ronovsky M, Partonen T, Pollak DD: Anhedonic behavior in cryptochrome 2-deficient mice is paralleled by altered diurnal patterns of amygdala gene expression. Amino Acids 2015, 47:1367-77.

[47] Haque R, Ali FG, Biscoglia R, Abey J, Weller J, Klein D, luvone PM: CLOCK and NPAS2 have overlapping roles in the circadian oscillation of arylalkylamine $\mathrm{N}$-acetyltransferase mRNA in chicken cone photoreceptors. J Neurochem 2010, 113:1296-306.

[48] Hanming G: nuotrophils arthritis. Research Square 2021.

[49] Gao L, Chen H, Li C, Xiao Y, Yang D, Zhang M, Zhou D, Liu W, Wang A, Jin Y: ER stress activation impairs the expression of circadian clock and clock-controlled genes in NIH3T3 cells via an ATF4dependent mechanism. Cell Signal 2019, 57:89-101.

[50] Fan XF, Wang XR, Yuan GS, Wu DH, Hu LG, Xue F, Gong YS: [Effect of safflower injection on endoplasmic reticulum stress-induced apoptosts in rats with hypoxic pulmonary hypertension]. Zhongguo Ying Yong Sheng Li Xue Za Zhi 2012, 28:561-7.

[51] Mao SZ, Fan XF, Xue F, Chen R, Chen XY, Yuan GS, Hu LG, Liu SF, Gong YS: Intermedin modulates hypoxic pulmonary vascular remodeling by inhibiting pulmonary artery smooth muscle cell proliferation. Pulm Pharmacol Ther 2014, 27:1-9.

[52] Ruan W, Yuan X, Eltzschig HK: Circadian rhythm as a therapeutic target. Nat Rev Drug Discov 2021, 20:287-307.

[53] Noda M, Iwamoto I, Tabata H, Yamagata T, Ito H, Nagata KI: Role of Per3, a circadian clock gene, in embryonic development of mouse cerebral cortex. Sci Rep 2019, 9:5874.

[54] Rauschmeier R, Gustafsson C, Reinhardt A, N AG, Tortola L, Cansever D, Subramanian S, Taneja R, Rossner MJ, Sieweke MH, Greter M, Mansson R, Busslinger M, Kreslavsky T: Bhlhe40 and Bhlhe41 transcription factors regulate alveolar macrophage self-renewal and identity. EMBO J 2019, 38:e101233.

[55] Tong H, Liu X, Li T, Qiu W, Peng C, Shen B, Zhu Z: NR1D2 Accelerates Hepatocellular Carcinoma Progression by Driving the Epithelial-to-Mesenchymal Transition. Onco Targets Ther 2020, 13:3931-42.

[56] Keniry M, Pires MM, Mense S, Lefebvre C, Gan B, Justiano K, Lau YK, Hopkins B, Hodakoski C, Koujak S, Toole J, Fenton F, Calahan A, Califano A, DePinho RA, Maurer M, Parsons R: Survival factor NFIL3 restricts FOXO-induced gene expression in cancer. Genes Dev 2013, 27:916-27.

[57] Gachon F, Fonjallaz P, Damiola F, Gos P, Kodama T, Zakany J, Duboule D, Petit B, Tafti M, Schibler U: The loss of circadian PAR bZip transcription factors results in epilepsy. Genes Dev 2004, 18:1397-412.

[58] Yuan J, Guo J, Zhang M, Wang Q, Huang H, Chen Y: Upregulation of D site of albumin promoter binding protein in the brain of patients with intractable epilepsy. Mol Med Rep 2015, 11:2486-92. 